\title{
Effect of early surgery after hip fracture on mortality and complications: systematic review and meta-analysis
}

\author{
Nicole Simunovic MSc, P.J. Devereaux MD, Sheila Sprague MSc, Gordon H. Guyatt MSc MD, \\ Emil Schemitsch MD, Justin DeBeer MD, Mohit Bhandari PhD MD
}

Previously published at www.cmaj.ca

\section{ABSTRACT}

Background: Guidelines exist for the surgical treatment of hip fracture, but the effect of early surgery on mortality and other outcomes that are important for patients remains unclear. We conducted a systematic review and meta-analysis to determine the effect of early surgery on the risk of death and common postoperative complications among elderly patients with hip fracture.

Methods: We searched electronic databases (including MEDLINE and EMBASE), the archives of meetings of orthopedic associations and the bibliographies of relevant articles and questioned experts to identify prospective studies, published in any language, that evaluated the effects of early surgery in patients undergoing procedures for hip fracture. Two reviewers independently assessed methodologic quality and extracted relevant data. We pooled data by means of the DerSimonian and Laird random-effects model, which is based on the inverse variance method.

Results: We identified 1939 citations, of which 16 observational studies met our inclusion criteria. These studies had a total of 13478 patients for whom mortality data were complete (1764 total deaths). Based on the five studies that reported adjusted risk of death (4208 patients, 721 deaths), irrespective of the cut-off for delay (24, 48 or 72 hours), earlier surgery (i.e., within the cut-off time) was associated with a significant reduction in mortality (relative risk [RR] $0.81,95 \%$ confidence interval $[\mathrm{Cl}] 0.68-0.96, p=0.01$ ). Unadjusted data indicated that earlier surgery also reduced in-hospital pneumonia (RR $0.59,95 \% \mathrm{Cl} 0.37-0.93, p=0.02$ ) and pressure sores (RR $0.48,95 \% \mathrm{Cl} 0.34-0.69, p<0.001$ ).

Interpretation: Earlier surgery was associated with a lower risk of death and lower rates of postoperative pneumonia and pressure sores among elderly patients with hip fracture. These results suggest that reducing delays may reduce mortality and complications.

$\mathrm{A}$ mong elderly patients, hip fracture is associated with a one-year mortality rate ranging from $14 \%$ to $36 \%^{1}$ and also with profound temporary and sometimes permanent impairment of independence and quality of life. ${ }^{2}$ As the elderly population increases, the annual number of hip fractures globally is expected to exceed 7 million over the next 40 to 50 years.

Current guidelines ${ }^{4}$ indicate that surgery for hip fracture should be performed within 24 hours of injury, as earlier surgery has been associated with better functional outcome, shorter hospital stay, shorter duration of pain and lower rates of nonunion, postoperative complications and mortality. ${ }^{5-11}$

Proponents of early treatment argue that this approach minimizes the length of time a patient is confined to bed rest, thereby reducing the risk of associated complications, such as pressure sores, deep vein thrombosis and urinary tract infections. ${ }^{8}$ However, those favouring a delay believe it provides the opportunity to optimize patients' medical status, thereby decreasing the risk of perioperative complications. ${ }^{8}$ A further challenge to resolving the debate is the lack of an accepted definition of early surgery. ${ }^{12}$ Uncertainty exists about whether 24,48 or 72 hours, or a longer period, should be considered to represent an "unacceptable delay" for hip fracture surgery.

We undertook a systematic review and meta-analysis to inform this debate. More specifically, we addressed the following question: Among patients 60 years of age or older who underwent surgery for hip fracture, what was the effect of early surgery, relative to delayed surgery, on all-cause mortality and postoperative complications?

\section{Methods}

\section{Eligibility criteria}

Studies fulfilling the following criteria were eligible for inclusion: target population consisting of patients 60 years of age or older who underwent surgery for a low-energy hip fracture, evaluation of preoperative surgical delay, consideration of allcause mortality as an outcome and prospective design. We imposed no language restrictions.

From the Department of Clinical Epidemiology and Biostatistics (Simunovic, Devereaux, Sprague, Guyatt, Bhandari) and the Department of Surgery (Sprague, DeBeer, Bhandari), Division of Orthopaedic Surgery, McMaster University, Hamilton, Ont.; and the Department of Surgery, Division of Orthopaedic Surgery (Schemitsch), University of Toronto, Toronto, Ont.

CMAJ 2010. DOI:10.1503/cmaj.092220 


\section{Identification of studies}

We used multiple strategies to identify potentially eligible studies. With the help of a professional librarian, we searched the electronic databases MEDLINE and EMBASE for relevant articles in any language that were published up to and including Feb. 8, 2008. The complete search strategies are shown in Appendix 1 (available at www.cmaj.ca/cgi/content /full/cmaj.092220/DC1). One reviewer (N.S.) also handsearched the archives of annual meetings of the Orthopaedic Trauma Association (1996-2007), the International Society of Orthopaedic Surgery and Traumatology (2003-2007), the Canadian Orthopaedic Association (2006-2007), the European Federation of National Associations of Orthopaedics and Traumatology (2005-2007), the Mid-America Orthopaedic Association (2005-2007), the Piedmont Orthopedic Society (2005-2007), the Association of Bone and Joint Surgeons (2005-2007) and the American Academy of Orthopaedic Surgeons (2006-2007) for any relevant unpublished literature. Additional strategies to identify studies included consultation with experts, a manual review of the reference lists of articles that fulfilled our eligibility criteria and use of the "related articles" feature in PubMed for any study that met our criteria.

\section{Screening and assessment of eligibility}

One reviewer (N.S.) screened the titles and abstracts of the studies from the electronic search to identify all citations that might contain the comparison of interest. Two review-

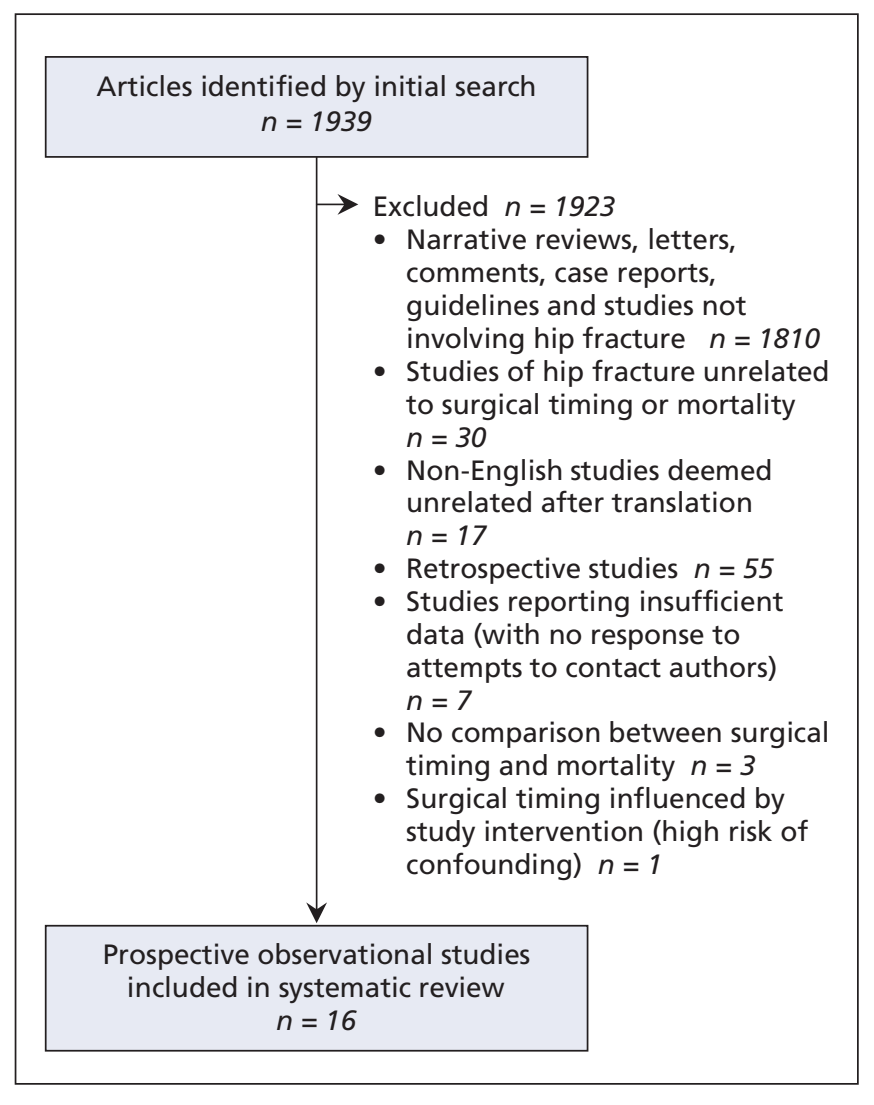

Figure 1: Flow of studies through the systematic review. ers (N.S., S.S.) independently evaluated these studies, as well as studies identified by hand-searching of reference lists and abstracts from meetings, to determine final inclusion (Figure 1).

Disagreements were resolved through a consensus process that required the reviewers to discuss the rationale for their decisions and come to an agreement. We recruited additional reviewers with an epidemiologic background and competence in various languages to apply the eligibility criteria to all non-English papers (one article in French, eight in German, three in Dutch and two in Hebrew). Study selection was not blinded, as blinding has been shown to have no significant statistical or clinical effect on the final results of systematic reviews. ${ }^{13}$

\section{Assessment of methodologic quality}

Two reviewers, both with methodologic expertise (N.S., S.S.) and one with content expertise (S.S.), independently graded the methodologic quality of each included study using an adapted version of the Newcastle-Ottawa Scale for Cohort Studies. ${ }^{14}$ This scale, which is intended to assess for selection and attrition bias, grades the reporting of studies on the basis of the selection, applicability and comparability of study groups; whether ascertainment of the exposure and outcome of interest was biased; and the adequacy of follow-up (ideally > 80\%). ${ }^{14}$ We deemed one item ("demonstration that outcome of interest was not present at start of study") irrelevant and removed it from the quality assessment because our primary outcome, all-cause mortality, was unequivocal. As a result, the maximum score was 8 , and the minimum score was 0 . We specified a priori that a score of 7 or more indicated high methodologic quality, a score of 5 or 6 indicated moderate quality, and a score of 4 or less indicated low quality. The reviewers resolved discrepancies for each item through discussion and, when necessary, re-evaluation of the study methodology until they reached consensus.

\section{Extraction of data}

When data for a study were unclear or missing from the article or abstract, we attempted to contact the authors. For all but one of the included studies, data had been collected prospectively for the purpose of answering a specific research question. The exception ${ }^{15}$ was a study that used prospectively collected data that had previously been entered into a hospital database. We confirmed with the authors of that study that the data had been collected accurately and consecutively for every eligible patient who had presented to two teaching hospitals, an approach that would have minimized the potential for selection bias.

\section{Assessment of agreement}

We used the $\kappa$ (kappa) statistic to examine the extent of agreement between the individuals who determined study eligibility. We used the intraclass correlation coefficient to evaluate interobserver agreement in methodologic quality scores. We chose an a priori criterion of $\kappa \geq 0.65$ to indicate adequate agreement. 


\section{Statistical analysis}

We included in our primary meta-analysis those studies that adjusted the mortality results for potentially confounding variables at any follow-up time. We included in our secondary meta-analyses studies with unadjusted estimates of mortality and postoperative complications.

When frequency data were available, we calculated the relative risk (RR) and 95\% confidence interval [CI] for the primary outcome (all-cause mortality) between the groups who underwent early and delayed surgery as assessed in hospital or at 30 days, at three to six months, and at one year. We grouped the data for in-hospital and 30-day follow-up on the basis of a sensitivity analysis that showed no statistical difference in mortality rates between follow-up assessments at these times. We considered adjusted estimates of mortality appropriate for the primary analysis if the authors had adjusted for at least patient age and type or severity of illness.

In the absence of frequency data, we collected the reported $\mathrm{RR}$, odds ratio or hazard ratio. We converted odds ratios and hazard ratios to RRs using the methods proposed by Zhang and $\mathrm{Yu}^{16}$ or the following formula: relative risk = $1-\mathrm{e}^{\text {hazard ratio* } \ln \left(1-P_{0}\right) / P_{0}}$, where $P_{0}$ is the outcome incidence in the non-exposed group.

Where appropriate, we pooled the outcome measures using the random-effects model of DerSimonian and Laird, ${ }^{17}$ which is based on the inverse variance method. We weighted all pooled estimates by study size. We quantified heterogeneity between studies using the $I^{2}$ statistic, ${ }^{18}$ which represents the percentage of total variation across trials that is due to heterogeneity rather than to chance. ${ }^{19}$

To assess publication bias, we constructed funnel plots to examine sample size versus exposure effect across included studies. This method plots the magnitude of the exposure effect relative to the weight of the study.

\section{Evaluation of heterogeneity}

Given the potential for heterogeneity in effect sizes, we performed stratified analyses and used a statistical test of interaction $^{20}$ to evaluate the extent to which subgroup results differed from each other. We hypothesized that heterogeneity might be due to differences in the reasons for surgical delay (administrative or non-administrative), the cut-off time used to define a delay (e.g., 24 v. 72 hours), the length of follow-up (e.g., 30 days v. one year), the date of publication of the study (before the year 2000 or in the year 2000 or later) or methodologic features (low quality v. moderate or high quality).

To control for multiple testing and inflation of type I error, we defined a significant difference between subgroups as $p<$ 0.01 . We considered $I^{2}<25 \%$ to indicate low heterogeneity and $I^{2}>75 \%$ to indicate high heterogeneity. ${ }^{21}$ Tests of significance for treatment effects were two-tailed, and a $p$ value of less than 0.05 was considered significant.

\section{Results}

\section{Studies included}

Our literature search identified 1939 potentially relevant citations: 1908 from the electronic search, 22 from hand searches of the articles that remained after initial screening $(n=98)$ and nine abstracts. Sixteen of these citations proved eligible for inclusion ${ }^{8,12,15,22-34}$ (Figure 1). Two studies were possible duplicates, but we included both in our analysis because one assessed long-term mortality ${ }^{24}$ and the other assessed shortterm mortality. ${ }^{25}$ The weighted $\kappa$ for overall agreement between reviewers for the final eligibility decision was 0.85 (95\% CI 0.47-1.00).

\section{Study characteristics}

The sample sizes of the included studies ranged from 65 to 3628 patients (Table 1). The cut-off times for operative delay were 24 hours, ${ }^{8,12,15,22,23,27,28,30,33} 48$ hours, ${ }^{24,25,29,31,32} 72$ hours $^{34}$ and five days. ${ }^{26}$ The preoperative interval was recorded from the time of injury to surgery in five studies ${ }^{12,15,25,28,29}$ and from the time of admission to surgery in the remaining 11 studies. Eight studies reported the reasons for surgical delay, the most common being the unavailability of an operating room and/or surgical personnel, ${ }^{12,24,25,27,31,32,34}$ and investigation and stabilization of the patient's preoperative medical condition. . $2,27,31,32,34^{2}$

\section{Study quality}

We judged four studies ${ }^{8,32-34}$ to be of high methodologic quality, five studies ${ }^{15,22,24,27,31}$ to be of moderate quality and the remaining seven studies ${ }^{12,23,25,26,28-30}$ to be of low quality (Table 1). Agreement between reviewers in the assessment of study quality was excellent (intraclass correlation coefficient 0.92 , 95\% CI 0.75-0.97).

\section{Mortality}

Of the 14171 patients analyzed in all 16 studies, complete mortality data were available for 13478 . In five studies $(n=$ 4208 patients), the researchers computed adjusted odds ratios or hazard ratios for mortality at 30 days, ${ }^{32}$ six months ${ }^{8}$ or one year $^{27,33,34}$ ( 721 total deaths) by means of a multivariable logistic regression model or multivariable Cox proportional hazards model. Those five studies most commonly adjusted for American Anesthetists Society score (a measure of a patient's fitness for surgery), age and sex. On the basis of the pooled adjusted estimates, early surgery was associated with a $19 \%$ risk reduction in all-cause mortality, irrespective of the time of the outcome assessment (RR 0.81, 95\% CI 0.68-0.96, $p=$ $0.01, I^{2}=0 \%$ ).

All 16 studies provided unadjusted estimates of mortality. The unadjusted estimates also suggested that early surgery significantly reduced the risk of one-year mortality by $45 \%$ (RR $0.55,95 \%$ CI $0.40-0.75, p<0.001, I^{2}=71 \%$; Figure 2). Heterogeneity in the unadjusted one-year mortality rate could not be explained by the reason for surgical delay, the cut-off for delay, study quality, date of publication or length of follow-up. Funnel plots showed no evidence of publication bias.

Time to surgery did not significantly affect mortality at 30 days $\left(n=3485\right.$; RR $0.90,95 \%$ CI $0.71-1.13, p=0.86, I^{2}=$ $0 \%)$ or at three to six months $(n=1650$ patients; RR 0.87 , 95\% CI 0.44-1.72, $p=0.68, I^{2}=87 \%$ ) (Figure 2). However, the removal of a single study evaluating only medically ill patients ${ }^{23}$ removed most of the between-study differences and 


\section{RESEARCH}

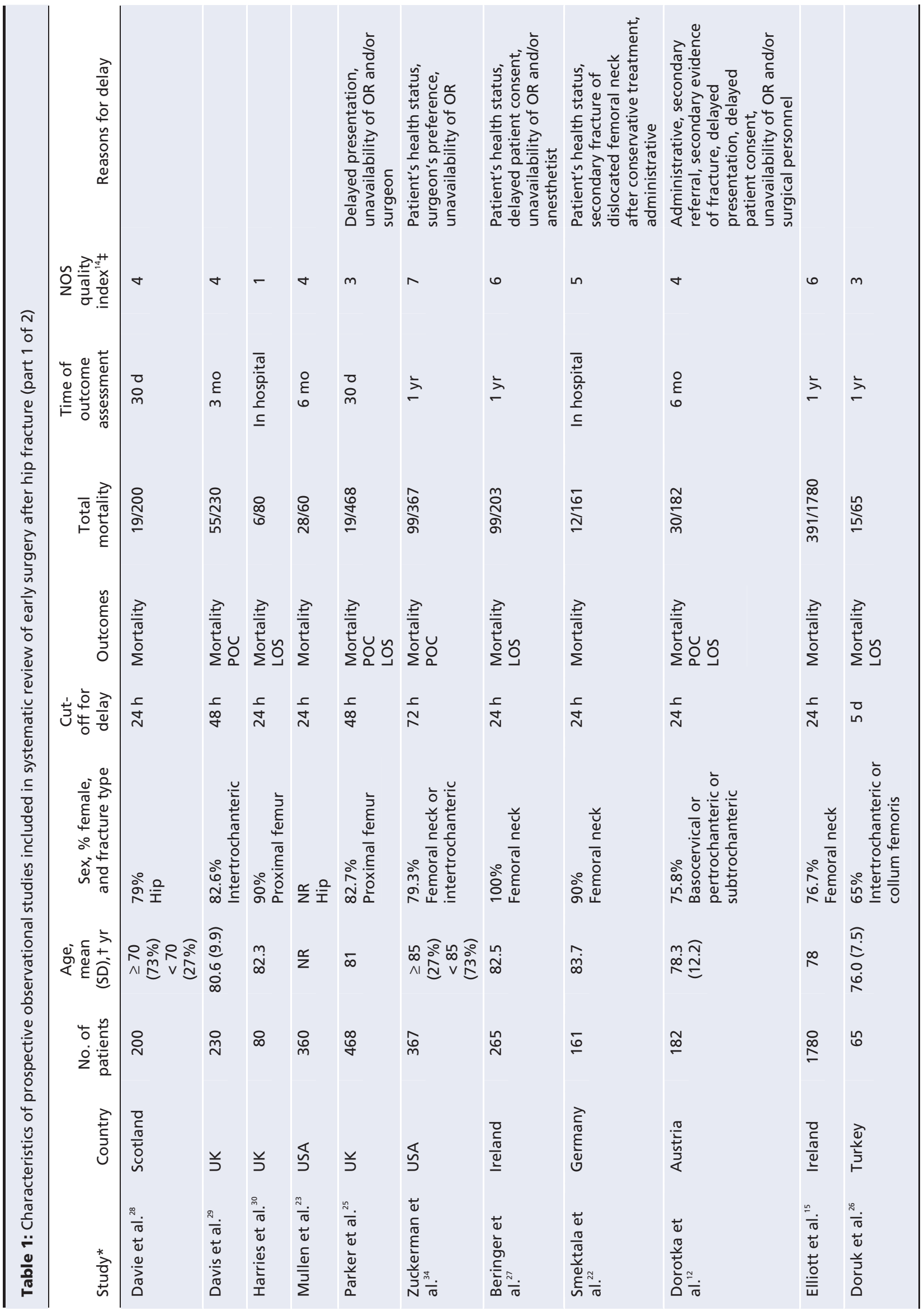




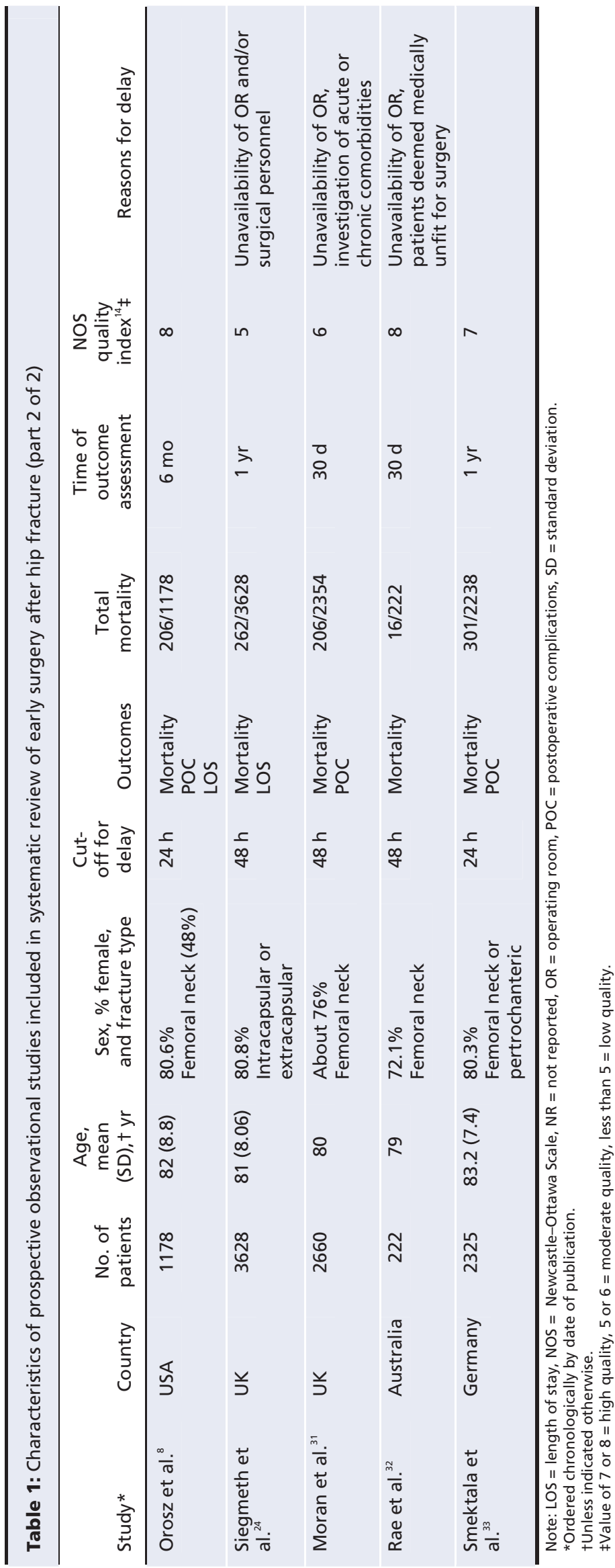

resulted in a significant benefit at three to six months with earlier surgery $(n=1590$ patients, RR $0.66,95 \%$ CI $0.50-0.88, p=$ $0.005, I^{2}=7 \%$ ).

\section{Postoperative complications}

Four studies ${ }^{25,29,31,33}$ reported on the number of individual postoperative complications for a total of 5377 patients. These data were not adjusted for potentially confounding surgical factors. Two of the studies ${ }^{25,33}$ evaluated pneumonia $(n=2793$ patients, 101 total events) and demonstrated an overall unadjusted risk reduction of $41 \%$ among patients who underwent early surgery $(<24$ or 48 hours) relative to those whose surgery was delayed (RR $0.59,95 \%$ CI $0.37-0.93, p=$ $0.02, I^{2}=0 \%$; Figure 3 ). In three studies, ${ }^{25,29,33}$ early surgery was associated with a $52 \%$ reduction in the risk of pressure sores, on the basis of unadjusted data $(n=3023$ patients, 174 total events) (RR $0.48,95 \% \mathrm{CI}$ $0.34-0.69, p<0.001, I^{2}=0 \%$; Figure 3).

Two of the studies evaluated deep vein thrombosis $^{31,33}$ ( $n=4679$ patients, 56 total events), and two studies examined pulmonary embolism ${ }^{25,31}$ ( $n=2822$ patients, 40 total events). Preoperative surgical delay did not significantly affect the unadjusted incidence of either of these complications (Figure 3).

\section{Interpretation}

Our primary meta-analysis suggested that early surgical treatment of hip fracture $(<24,<48$ or $<72$ hours) was associated with a significant reduction in mortality. Earlier surgery was also associated with a reduced risk of pneumonia and pressure sores. Some authors have argued that an important contributing factor (if not the main factor) affecting mortality in patients whose surgery is delayed is that these patients tend to be sicker on admission, and are therefore more likely to die, than those who undergo surgery immediately. ${ }^{35,36}$ Nonetheless, our primary meta-analysis showed a significant influence of surgical delay on mortality, even after adjustment for confounding preoperative factors. Further study is needed to examine the differences in outcomes between medically fit and medically unfit patients and to determine whether increasing the availability of surgical resources will yield cost-effective benefits.

A recent systematic review reported that 


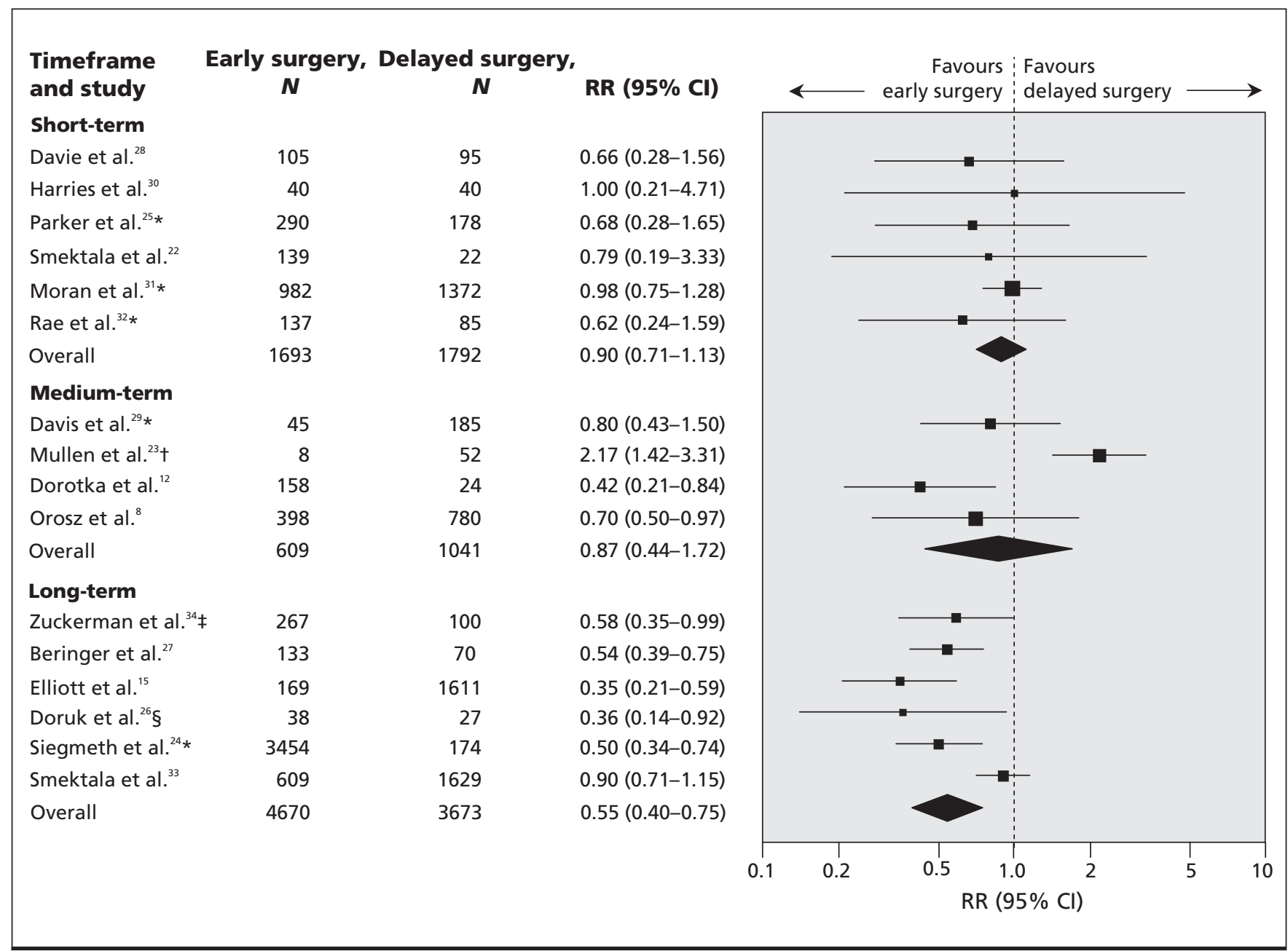

Figure 2: Stratified analysis by time of death. Forest plot of unadjusted relative risks for the effect of early compared with delayed surgery for hip fracture on all-cause mortality assessed in hospital or at 30 days (short-term), at three to six months (medium-term) or at one year (long-term) (random-effects model based on inverse variance method). Studies used a cut-off for delay of 24 hours, except as indicated otherwise. *Study used a cut-off of 48 hours for delay. †Data based on patients who had medical illness in combination with hip fracture. ‡Study used a cut-off of 72 hours for delay. §Study used a cut-off of 5 days for delay. $\mathrm{Cl}=$ confidence interval, $\mathrm{RR}=$ relative risk.

a delay of more than 48 hours was associated with increased mortality among patients with hip fracture. ${ }^{37}$ However, that systematic review had methodologic limitations, including a restriction to English-language articles and inclusion of retrospective studies, which are often more prone to bias (e.g., recall bias, selection bias) and which may therefore lead to overestimation of treatment effects. ${ }^{38}$ Our wider eligibility criteria and search identified 11 prospective observational studies (10 in English and one in German) additional to those in the study by Shiga and colleagues. ${ }^{37}$ Using the selection criteria that were used by Shiga and colleagues, ${ }^{37}$ we identified four additional prospective studies.

\section{Limitations}

Our meta-analysis had some limitations. It is likely that our estimates of postoperative complications were subject to publication bias because we restricted data collection for that analysis to the 16 studies with data on mortality rates for patients undergoing early or delayed surgery. Our findings that early surgery may reduce the risk of pneumonia and pressure sores should therefore be interpreted with caution. In addition, we identified unexplained heterogeneity in our analysis of the unadjusted one-year mortality outcome.

The most notable limitation of this review was its restriction to observational studies, which reflects the state of current evidence. Observational studies are prone to selection, performance, attrition and detection bias. Unadjusted analyses are certainly confounded, and although we used adjusted ratios for our primary meta-analysis, the results may still be subject to confounding bias where our study may be missing other unknown or unmeasured factors potentially relevant to prognosis for a patient with hip fracture. These factors may limit the conclusions.

\section{Conclusion}

On the basis of current evidence, surgery conducted before 24-72 hours is associated with lower mortality and lower rates of certain postoperative complications among elderly 


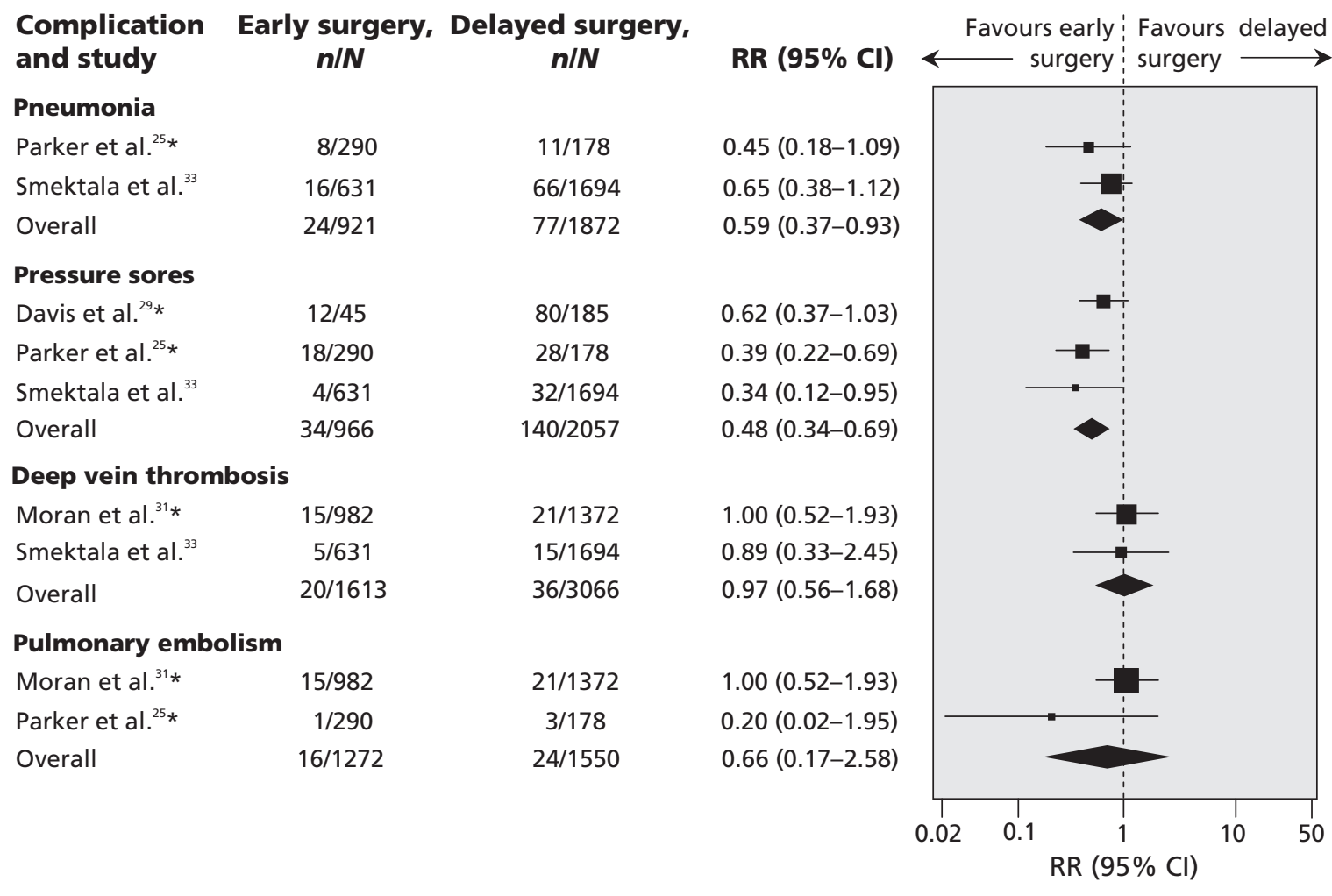

Figure 3: Forest plot of unadjusted relative risks for the effect of preoperative timing on specific postoperative complications assessed in hospital (random-effects model). Studies used a cut-off for delay of 24 hours, except as indicated otherwise. *Study used a cut-off of 48 hours for delay. $\mathrm{Cl}=$ confidence interval, $\mathrm{RR}=$ risk ratio.

patients with hip fracture. When potential confounding preoperative factors are taken into account, this effect is not as large, but its direction is maintained. Given the challenges in interpreting observational data, there is a need for additional well-designed prospective studies or a randomized trial to offer clear insights into the effects of early surgery in this patient population.

This article has been peer reviewed.

Competing interests: Mohit Bhandari has received research grants from Smith and Nephew, Stryker, DePuy, Zimmer, the Canadian Institutes for Health Research, the Osteosynthesis and Trauma Care Foundation, the AO (Arbeitsgemeinschaft für Osteosynthesefragen) Foundation, the National Institutes of Health, the US Department of Defense and the Orthopedic Trauma Association. None declared for Nicole Simunovic, P.J. Devereaux, Sheila Sprague, Gordon Guyatt, Emil Schemitsch and Justin DeBeer.

Contributors: Nicole Simunovic contributed substantially to the conception and design of the study and to the acquisition, analysis and interpretation of the data; she also drafted the manuscript. P.J. Devereaux, Gordon Guyatt, Emil Schemitsch and Justin DeBeer contributed substantially to the conception and design of the study and revised the article for important intellectual content. Sheila Sprague contributed substantially to the acquisition of the data and revised the article for important intellectual content. Mohit Bhandari contributed substantially to the conception and design of the study and the interpretation of the data and revised the article for important intellectual content. All authors gave final approval of the version to be published.

Funding: No external funding was received for this paper.

\section{REFERENCES}

1. Zuckerman JD. Hip fracture. N Engl J Med 1996;334:1519-25.

2. Johnell O, Kanis JA. An estimate of the worldwide prevalence, mortality and disability associated with hip fracture. Osteoporos Int 2004;15:897-902.

3. Parker M, Johansen A. Hip fracture. BMJ 2006;333:27-30.

4. Hip \& pelvis (acute \& chronic) [guideline]. Corpus Christi (TX): Work Loss Data Institute; 2008. Available: www.guideline.gov/content.aspx?id=12672 (accessed 2010 June 14).

5. Bottle A, Aylin P. Mortality associated with delay in operation after hip fracture: observational study. BMJ 2006;332:947-51.

6. Grimes JP, Gregory PM, Noveck H, et al. The effects of time-to-surgery on mortality and morbidity in patients following hip fracture. Am J Med 2002;112:702-9.

7. Manninger J, Kazar G, Fekete G, et al. Significance of urgent (within 6h) internal fixation in the management of fractures of the neck of the femur. Injury 1989;20:101-5.

8. Orosz GM, Magaziner J, Hannan EL, et al. Association of timing of surgery for hip fracture and patient outcomes. JAMA 2004;291:1738-43.

9. Perez JV, Warwick DJ, Case CP, et al. Death after proximal femoral fracture - an autopsy study. Injury 1995;26:237-40.

10. Rogers FB, Shackford SR, Keller MS. Early fixation reduces morbidity and mortality in elderly patients with hip fractures from low-impact falls. J Trauma 1995;39:261-5.

11. Villar RN, Allen SM, Barnes SJ. Hip fractures in healthy patients: operative delay versus prognosis. BMJ (Clin Res Ed) 1986;293:1203-4.

12. Dorotka R, Schoechtner H, Buchinger W. [Influence of nocturnal surgery on mortality and complications in patients with hip fracture.] Unfallchirurg 2003;106:287-93. Article in German.

13. Berlin JA. Does blinding of readers affect the results of meta-analyses? University of Pennsylvania Meta-analysis Blinding Study Group. Lancet 1997;350:185-6.

14. Wells GA, Shea B, O'Connell D, et al. The Newcastle-Ottawa Scale (NOS) for assessing the quality of nonrandomised studies in meta-analyses. Ottawa (ON): Ottawa Health Research Institute. Available: www.ohri.ca/programs/clinical _epidemiology/oxford.htm (accessed 2010 June 1).

15. Elliott J, Beringer T, Kee F, et al. Predicting survival after treatment for fracture of the proximal femur and the effect of delays to surgery. J Clin Epidemiol 2003;56:788-95. 
16. Zhang J, Yu KF. What's the relative risk? A method of correcting the odds ratio in cohort studies of common outcomes. JAMA 1998;280:1690-1.

17. DerSimonian R, Laird N. Meta-analysis in clinical trials. Control Clin Trials 1986;7:177-88.

18. Higgins JPT, Thompson SG. Quantifying heterogeneity in a metaanalysis. Stat Med 2002;21:1539-58

19. Devereaux PJ, Beattie WS, Choi PTL, et al. How strong is the evidence for the use of perioperative $\beta$ blockers in non-cardiac surgery? Systematic review and metaanalysis of randomised controlled trials. BMJ 2005;331:313-21.

20. Altman DG, Bland JM. Interaction revisited: the difference between two estimates. BMJ 2003;326:219.

21. Higgins JPT, Green S, editors. Cochrane handbook for systematic reviews of interventions. Version 5. The Cochrane Collaboration; 2008. Available: www.cochrane -handbook.org (accessed 2010 June 1).

22. Smektala R, Wenning M, Luka M. Early surgery after hip para-articular femora fracture. Results of a prospective study of surgical timing in 161 elderly patients. Zentralbl Chir 2000;125:744-9.

23. Mullen JO, Mullen NL. Hip fracture mortality: a prospective, multifactorial study to predict and minimize death risk. Clin Orthop Relat Res 1992;280:214-22.

24. Siegmeth AW, Gurusamy K, Parker MJ. Delay to surgery prolongs hospital stay in patients with fractures of the proximal femur. J Bone Joint Surg Br 2005;87:1123-6.

25. Parker MJ, Pryor GA. The timing of surgery for proximal femoral fractures. $J$ Bone Joint Surg Br 1992;74:203-5.

26. Doruk H, Mas MR, Yildiz C, et al. The effect of the timing of hip fracture surgery on the activity of daily living and mortality in elderly. Arch Gerontol Geriat 2004;39:179-85.

27. Beringer TR, Crawford VL, Brown JG. Audit of surgical delay in relationship to outcome after proximal femoral fracture. Ulster Med J 1996;65:32-8.

28. Davie IT, MacRae WR, Malcolm-Smith NA. Anesthesia for the fractured hip Anesth Analg 1970;49:165-70.
29. Davis TR, Sher JL, Porter BB, et al. The timing of surgery for intertrochanteric femoral fractures. Injury 1988;19:244-6.

30. Harries DJ, Eastwood H. Proximal femoral fractures in the elderly: Does operative delay for medical reasons affect short-term outcome? Age Ageing 1991;20:41-4.

31. Moran CG, Wenn RT, Sikand M, et al. Early mortality after hip fracture: Is delay before surgery important? J Bone Joint Surg Am 2005;87:483-9.

32. Rae HC, Harris IA, McEvoy L, et al. Delay to surgery and mortality after hip fracture. Aust N Z J Surg 2007;77:889-91.

33. Smektala R, Endres H, Dasch B, et al. The effect of time-to-surgery on outcome in elderly patients with proximal femoral fractures. BMC Musculoskelet Disord 2008; 9:171.

34. Zuckerman JD, Skovron ML, Koval KJ, et al. Postoperative complications and mortality associated with operative delay in older patients who have a fracture of the hip. J Bone Joint Surg Am 1995;77:1551-6.

35. Hamlet WP, Lieberman JR, Freedman EL, et al. Influence of health status and the timing of surgery on mortality in hip fracture patients. Am J Orthop 1997;26: 621-7.

36. Hardin GT. Timing of fracture fixation: a review. Orthop Rev 1990;19:861-7.

37. Shiga T, Wajima Z, Ohe Y. Is operative delay associated with increased mortality of hip fracture patients? Systematic review, meta-analysis, and meta-regression. Can J Anaesth 2008;55:146-54.

38. Hulley SB, Cummings SR, Browner WS, et al. Designing clinical research. 3rd ed. Philadelphia (PA): Lippincott Williams and Wilkins; 2007.

Correspondence to: Nicole Simunovic, CLARITY Orthopaedic

Research, 293 Wellington St. N, Suite 110, Hamilton ON

L8L 8E7; simunon@mcmaster.ca

\section{ECZEMA FLARES ARE ALWAYS WAITING TO ATTACK.}

